\title{
Effect of human papillomavirus infection on the immune system and its role in the course of cervical cancer (Review)
}

\author{
DAN SONG ${ }^{*}, \mathrm{HONG} \mathrm{LI}^{1 *}, \mathrm{HAIBO} \mathrm{LI}^{1}$ and JIANRONG DAI ${ }^{2}$ \\ ${ }^{1}$ Center for Reproduction and Genetics; ${ }^{2}$ Department of Gynecology, Suzhou Municipal Hospital, \\ Nanjing Medical University Affiliated Suzhou Hospital, Suzhou, Jiangsu 215002, P.R. China
}

Received July 30, 2014; Accepted April 13, 2015

DOI: 10.3892/ol.2015.3295

\begin{abstract}
Human papillomavirus (HPV) is widely known as a cause of cervical intraepithelial neoplasia (CIN) and cervical cancer. The mechanisms involved have been studied by numerous studies. The integration of the virus genome into the host cells results in the abnormal regulation of cell cycle control. HPV can also induce immune evasion of the infected cells, which enable the virus to be undetectable for long periods of time. The induction of immunotolerance of the host's immune system by the persistent infection of HPV is one of the most important mechanisms for cervical lesions. The present review elaborates on the roles of several types of immune cells, such as macrophages and natural killer cells, which are classified as innate immune cells, and dendritic cells (DCs), cluster of differentiation (CD) $4^{+} / \mathrm{CD} 8^{+} \mathrm{T}$ cells and regulatory $\mathrm{T}$ cells, which are classified as adaptive immune cells. HPV infection could effect the differentiation of these immune cells in a unique way, resulting in the host's immune tolerance to the infection. The immune system modifications induced by HPV infection include tumor-associated macrophage differentiation, a compromised cellular immune response, an abnormal imbalance between type 1 T-helper cells (Th1) and Th2 cells, regulatory T cell infiltration, and downregulated DC activation and maturation. To date, numerous types of preventative vaccines have been created to slow down carcinogenesis. Immune response activation-based therapeutic vaccine is becoming more and more attractive for the treatment of HPV-associated diseases.
\end{abstract}

Correspondence to: Dr Jianrong Dai, Department of Gynecology, Suzhou Municipal Hospital, Nanjing Medical University Affiliated Suzhou Hospital, 26 Daoqian Street, Suzhou, Jiangsu 215002, P.R. China

E-mail: dayle2008@sina.com

*Contributed equally

Key words: human papillomaviruses, dendritic cells, $\mathrm{T}$ cells, macrophages, regulatory $\mathrm{T}$ cells, cervical intraepithelial neoplasia, cervical cancer

\section{Contents}

1. Introduction

2. HPV infection regulating the host immune response

3. Immune cell changes in the immune response to hrHPV

4. Immune-related markers of CIN and cervical cancer

5. Preventive and therapeutic HPV vaccines

6. Conclusion

\section{Introduction}

Cervical cancer accounts for $15 \%$ of cancers in females aged $<65$ years old in developing countries (1). Long-term high-risk human papillomavirus (hrHPV) infection must be present, but is insufficient to cause cervical cancer by itself according to the International Agency for Research on Cancer (IARC). Certain other risk factors account for the development of cervical cancer, including an active sexual history, weakened immune system function and smoking (2). The induction of cervical carcinogenesis by persistent hrHPV infection is a multistep process, consisting of persistent infection, different stages of cervical intraepithelial neoplasia (CIN) lesions and ultimately, cervical cancer (3). Although persistent hrHPV infection is an important prerequisite for CIN, the vast majority (90\%) of viruses have been cleared by the hosts' immune system, without medical intervention, as observed on the three-year follow-up examination, and only $1-2 \%$ of the remaining $10 \%$ become chronic resulting in cervical cancer (3).

To date, $>180$ types of HPV are known to exist (4). HPV16, $18,31,33,35,39,45,51,52,56,58,59,68$ and 69 are classified as the hrHPVs (5). HPV6, 11, 40, 42, 54, 55, 61, 62, 64, 71, 72, 81,83 and 84 are classified as the low-risk HPVs (6). HPV16 and 18 are the most common types, accounting for $~ 70 \%$ of cervical cancers around the world (7).

HrHPV infection starts with the contact of the virus with the basement membrane, which is often exposed by micro-abrasions on the cervical surface. Suitable receptors increase the probability of hrHPV infection. Studies have found several receptors involved in this process. Interaction between the virus' capsid protein and the cell receptors promotes virus capsid conformational changes, thus aiding the cell entry process (8). Tissue-specific heparin sulfate proteoglycan (HSPG), as one of the members of the glycosaminoglycan 
family, is a receptor of major capsid protein L1 of $\operatorname{HPV}(9,10)$. HSPG could aid HPV16 in binding to the extracellular matrix via laminin-332 (11).

HPV DNA is double-stranded, containing 7,900 base pairs, which are arranged in a circle (12). The genome consists of 8 open reading frames, 6 early genes (E1, E2, E4, E5, E6 and E7) encoding the early protein and 2 late genes (L1 and L2) encoding the late protein. E1 protein aids in viral replication utilizing the host replication machinery. E5, E6 and E7 proteins are considered to be associated with virus immune evasion. The product of E5 could downregulate the histocompatibility leucocyte antigen (HLA) expression of the infected cells, facilitating virus immune escape $(13,14)$. E6 and E7 are considered as tumorigenic genes; their products could bind to tumor suppressor protein p53 and arrest cell cycle, impairing the infected cells apoptosis and enhancing their transformation (15). The L1 and L2 proteins are responsible for forming the structural components of the viral capsid. E2 exhibits a regulatory function for E6 and E7 transcription. HPV genome integration into the host genome would disrupt the regulatory function of E2, leading to the uncontrolled expression of E6 and E7, promoting the progression of the disruption of the normal cell cycle and carcinogenesis (16).

Furthermore, the expression of E6 and E7 is fundamental in hrHPV infection and CIN progression. The expression of these oncoproteins inhibits the immune system response to hrHPV and aids in the persistency of the infection. The oncoproteins could downregulate TLR9 expression, a virus DNA sensor, which is necessary to activate antigen-presenting cells (17), but they could also reduce the expression of transporter-associated antigen processing 1, blocking the activation of specific $\mathrm{T}$ lymphocytes. It has been confirmed that neoplastic cervical keratinocytes (KCs) expressing high levels of E6 and E7 oncoproteins could escape the attack from cytotoxic T cells (CTLs) (18).

\section{HPV infection regulating the host immune response}

According to a previous study, the incubation period of HPV infection is $\sim 10$ years. The immune response of the host plays a crucial role in the progression or regression of hrHPV infection of the uterine cervix. An effective immune response promotes spontaneous clearance of the virus, while a compromised immune response often starts up the pathological process, developing it into the higher grade (19). HrHPV infection causes several events, which are essential for CIN progression and carcinogenesis (Fig. 1).

HrHPV infection promotes immune cell migration to the dermis. In the squamous epidermis, macrophages, Langerhans cells (LC), KCs, T lymphocytes, dendritic cells (DC), natural killer cells (NK) and B lymphocytes play important roles during the immune response to infection. HrHPV infection could cause the immune system to become more tolerant to the infection, thus creating a microenvironment susceptible to further infection and facilitating CIN progression. The mechanisms that have been proposed and proved are as follows: Firstly, hrHPV remains silent for a long time; its duplication and assembly do not cause cytolysis or the cytopathic death of the host cells (20). Secondly, hrHPV inhibits interferon (IFN) synthesis through E6 and E7 oncoproteins interfering with IFN signaling pathways (21).
Thirdly, hrHPV infection induces regulatory T cell (Treg) infiltration and interleukin (IL)-10 or transforming growth factor $\beta$ (TGF- $\beta$ ) production. Fourthly, the infected cells express low levels of MHC class I, resulting in impaired CTL function (22). Fifthly, they could induce an accumulation of ineffective CD4 and CD8 T lymphocytes in stage II/III CINs (23).

Additionally, KCs are the major cell type in the epidermis, with certain immune functions, such as cytokine secretion. The compromised innate immune defense in KCs is an important reason for the evasion of the hrHPV infection from the immune response, ultimately resulting in persistent virus infection and the development into pre-neoplastic lesions (24).

Innate immunity affected by hrHPV infection. Usually, once the hrHPV contacts the mucosal epithelium, the innate immunity system mediated by the epithelial barrier starts to conflict with it. LCs are immature DCs, and in the transformation zone their numbers are significantly decreased. The mechanism proposed for this is the direct interaction of E7 with CCAAT/enhancer-binding protein $\beta$, a transcription factor of chemokine (C-C motif) ligand 20 (CCL20) (which has a decisive role in the migration of LC precursors into the epidermis), thereby inhibiting the transcription of CCL20 and thus hindering LC recruitment (25).

Toll-like receptors (TLRs) are an important type of pattern recognition receptor located at the endolysosomal compartments, sensing the bacteria/virus and triggering the associated innate immune response. Several of these receptors have been investigated. HrHPV18 E6 and E7 downregulate TLR9 (specific for the nucleic acid) expression at the infection site, an important strategy for its escaping from immunosurveillance (26). In contrast to TLR9, the TLR3/5/8 pathways are activated in hrHPV-infected KCs. High expression levels of TLR8 in cervical cancer cells are associated with the upregulated expression of B-cell lymphoma-2 and vascular endothelial growth factor in these cells (27). A previous study found that there is marked TLR4 expression in hrHPV-positive cells and that its expression is associated with the virus type, as well as the histopathological grade. Higher TLR4 expression is found in $\mathrm{HPV} 16^{+}$cells compared with HPV18 ${ }^{+}$cells, and in cervical cancer compared with CIN. Additionally, it has been demonstrated that TLR4 overexpression is correlated with the apoptotic resistance of the HPV-infected cells (28).

It is known that tumor-associated macrophages, also termed M2 macrophages, can promote cancer cell proliferation and migration, angiogenesis and the restriction of immune defenses (29). During cervical lesion progression, the number of M2 macrophages is significantly increased (30). The aggregation of M2 is a key event for the pathological process of carcinogenesis.

HrHPV infection compromises NK cell activation. NK cells predominate at the initial stage of the infection and in the low-grade lesions. Levels of NK-activating receptors, such as NKp30, NKp45, NKp46, NKG2D and NKp80, are significantly decreased in HPV16 cervical cancer. These receptors are closely associated with the low cytotoxic activity of NK cells, facilitating lesion progression and carcinogenesis (31). hrHPV-infected tissues or cervical cancer cells could obtain their immune evasion abilities by making NK cells more tolerant to the hrHPV infection and cervical carcinogenesis (32). 


\section{long time period}

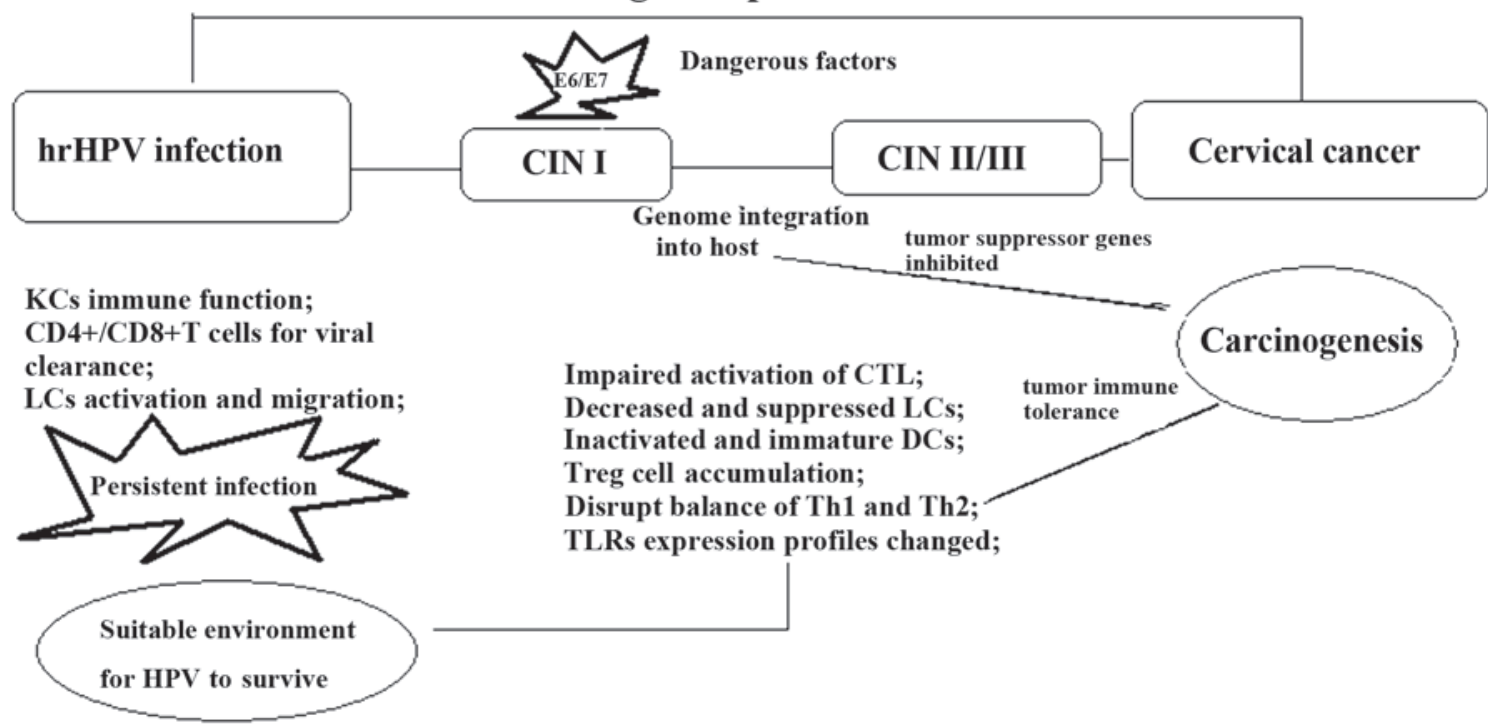

Figure 1. There are several stages from HPV infection to cervical cancer. Immune system modification is the most important factor to promote lesion progression. Genome integration would initiate immune cell activation and differentiation profiles changes. These changes are characterized by the inactivated $\mathrm{CD}^{+} / \mathrm{CD}^{+} \mathrm{T}$ cells, Treg cell upregulation, M2 cell generation, the immature DCs and reduced anti-inflammatory cytokine infiltration. hrHPV, high-risk human papilloma virus; CIN, cervical intraepithelial neoplasia; CD, cluster of differentiation; LC, Langerhans cell; CTL, cytotoxic T cell; DC, dendritic cell; Th, T-helper cell; TLR, toll-like receptor.
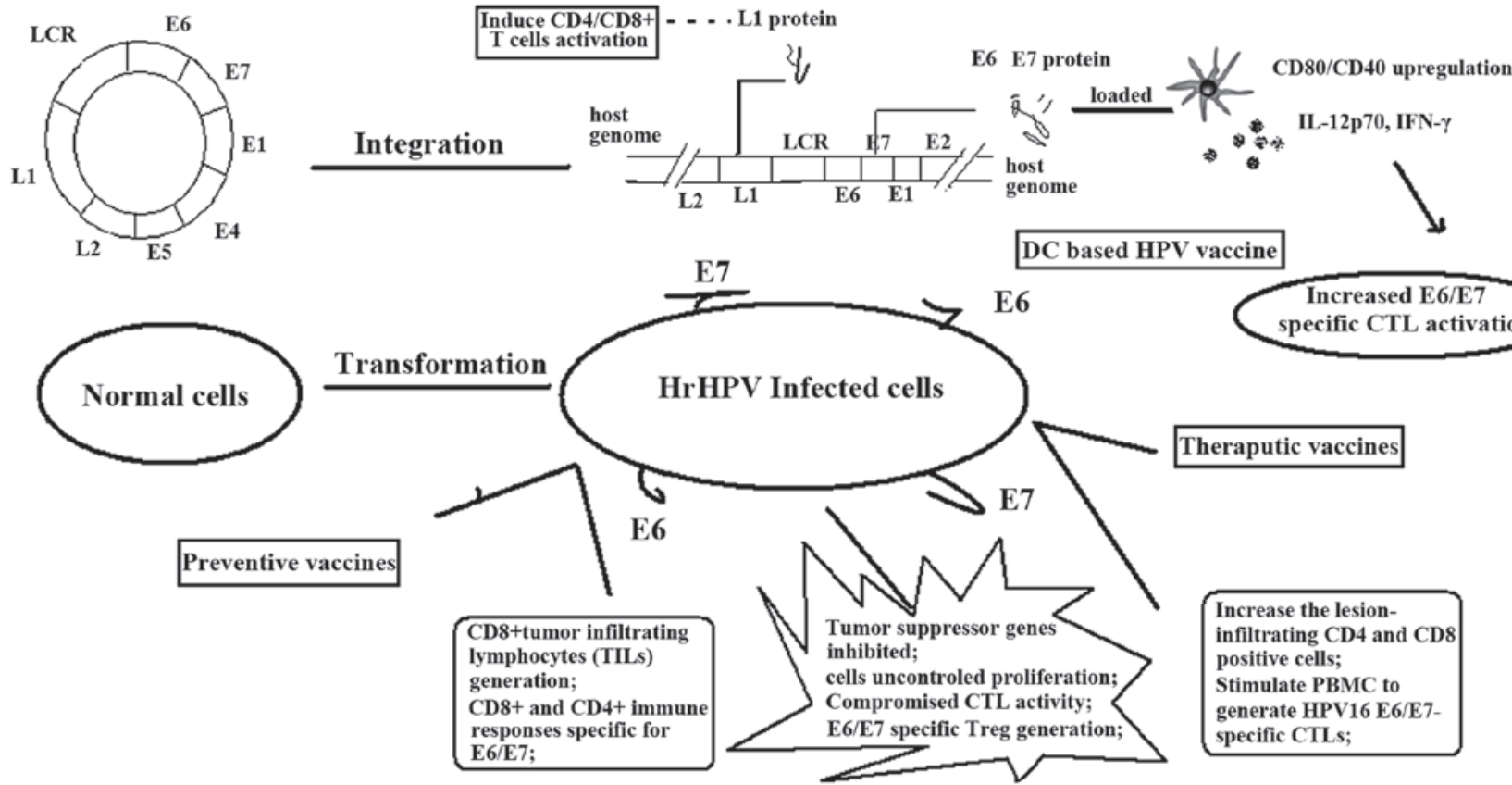

Figure 2. Mechanisms for vaccines in the prevention and therapy of HPV infection and CIN progression. Vaccines aim to improve the HPV oncoprotein-specific immune response, such as cytokine production, CTL initiation and immune system modification, contributing to the treatment of every stage from HPV infection to CIN to cervical cancer. CD, cluster of differentiation; IL, interleukin; IFN, interferon; DC, dendritic cell; hrHPV, high-risk human papilloma virus; CTL, cytotoxic T cell; PBMC, peripheral blood mononuclear cell; Treg, regulatory T cell; LCR, long control region.

\section{Immune cell changes in the immune response to hrHPV}

Compromised adaptive immunity is the foundation for the progression from hrHPV infection to cervical cancer. There are different immune cell profiles for the different stages of the disease progression in CIN and carcinogenesis. The changes and modifications induced by hrHPV infection are involved in hrHPV infection adapting the immune system to create a suitable microenvironment for persistent infection and lesion progression.

HPV infection induces compromised $T$ cell activation. $\mathrm{T}$ cell activation is important in hrHPV infection. It has been proven that in patients with HIV infection, compromised 
$\mathrm{CD}^{+} \mathrm{T}$ cells function as the inducer for HPV-associated cancer occurrence (20). For these patients, the induction of inefficient $\mathrm{CD}^{+} \mathrm{T}$ cells by hrHPV infection is the factor promoting CIN lesion progression (33). CTL is the main agent in cancer specific immunity, and it recognizes the antigens with the assistance of MHC/HLA class I. HPV16 E5 could suppress CTL through the downregulation of MHC/HLA class I expression, as aforementioned.

Distorted equilibrium between type 1 T-helper cells (Th1) and Th2 cell is another property of cellular immunity during hrHPV infection (34). There is a marked Th2 cytokine profile in intraepithelial and invasive cervical lesions. Increased Th2 cytokine (IL-10) and reduced Th1 cytokine (IFN- $\gamma$, IL-12, IL-2 and tumor necrosis factor- $\alpha$ ) levels have been detected in cervical exudates of $\mathrm{hrHPV}^{+}$patients. This indicated that the reduced $\mathrm{Th} 1$ response and increased $\mathrm{Th} 2$ response lead to cellular immunity suppression and cervical lesion progression $(35,36)$. Another study proved that the production of certain Th2 cytokines is also decreased during this process, indicating that the Th1 and Th2 phenotypes, but particularly the Th1 phenotype, may be suppressed by hrHPV infection. One of the mechanisms by which hrHPV regulates $\mathrm{T}$ cell activation is based on E6 and E7 oncoprotein expression, which would upregulate the expression of adhesion molecules, such as intercellular adhesion molecule 1, vascular cell adhesion molecule 1 and E-selectin (classical immunosuppressive molecules), in the infected cells (37).

Overall, compromised $\mathrm{T}$ cell activation and the distorted equilibrium between Th1 and Th2 presents hrHPV with the ability to escape from the human immune defense.

Compromised DC activation induced by hrHPV infection. DCs, as one of the important adaptive immune cells, recognize the special patterns of pathogens through TLRs and present the antigens to $\mathrm{T}$ cells through MHC molecules to initiate the antigen-specific immune response. A high level of stromal DCs is usually associated with a higher frequency of infection regression. Along with hrHPV infection, immature DCs usually prevent the activation of a correct immune response by CTLs and facilitate lesion progression (38). There is an extremely low distribution or complete absence of functional DCs in patients with cervical cancer. HrHPV E6-expressing cells or $\mathrm{hrHPV}^{+}$cancer cells can inhibit the differentiation of monocytes into fully functional DCs (39). A low percentage of plasmacytoid DCs in the peripheral blood is significantly associated with persistent hrHPV infection (40). Furthermore, it has been demonstrated that in $\mathrm{hrHPV}^{+}$patients, decreased expression levels of CD80 and CD86 in DCs have a positive correlation with increased CIN grades (41).

One of the mechanisms involved in the induction of compromised DC activation in hrHPV infection was proposed as the stimulation of programmed death 1 (PD-1)/PD-1 ligand (PD-L1) (CD279/CD274) pathway activation by chronic hrHPV infection in DCs. PD-1/PD-L1 activation is associated with impaired DC-mediated immunity to hrHPV infection, thus creating the immune tolerance microenvironment for persistent infection and CIN progression (41).

Interplay between Treg cells and hrHPV infection. Tregs $\left(\mathrm{CD}^{+} / \mathrm{CD} 25^{+} / \mathrm{Foxp}^{+} \mathrm{T}\right.$ cells) are the inducer of immune tolerance. There is increased Foxp $3^{+}$Treg infiltration following hrHPV infection. Recent studies have highlighted the role of hrHPV-specific Treg generation induced by hrHPV infection and CIN progression (42). Tregs specific for the E6 and E7 antigens have been detected in high-grade squamous intraepithelial lesion (HSIL)-infiltrating lymphocytes (43), and there are several mechanisms that contribute to their generation and recruitment. Firstly, improper activation of the immune response induced by hrHPV infection provides the possibility of the toleration of $\mathrm{T}$ cell generation. Secondly, the weakened innate immune function elicited by the infected KCs creates an immunosuppressive microenvironment, promoting HPV-specific Treg expansion (22).

The regulation of hrHPV infection and CIN progression by Treg cells is mainly based on cytokine production. For example, TGF- $\beta$ is produced by Treg, and its level has a positive correlation with lesion progression (36). IL-10 is another significant Treg-producing cytokine whose presence would decrease $\mathrm{CD}^{+} \mathrm{T}$ cell infiltration and increase the amount of intratumoral Foxp $3^{+}$Treg cells, a critical event for CIN lesion progression $(44,45)$. There is also a notable interaction between IL-10 and HPV-induced lesion progression (46). In hrHPV infected cells, E2 protein binds to the regulatory region of the human IL-10 gene (-2054 nt) and induces its expression; IL-10 can create an immune-tolerant microenvironment, providing the possibility of progressive cervical disease in turn (47).

\section{Immune-related markers of CIN and cervical cancer}

Immune markers (immune cells, cytokines, chemokines and soluble receptors) during persistent hrHPV infection and CIN progression have not been well studied (48). The percentage of Tregs is not only used as a marker of tumor immune status, but is also considered as a possible marker of immune destruction induced by hrHPV infection. Foxp3, as the only definitive marker of Tregs, exhibits gradually increased expression from CIN I to CIN III, which indicates that Foxp3 is involved in the development of cervical cancer (49). However, the exact mechanism of such requires further investigation.

High mobility group box 1 protein (HMGB1), a DNA-binding protein, is critical for host immune suppression. There is a direct correlation between HMGB1 expression and the malignant potential of CIN. A previous study also proved that high levels of HMGB1 expression can be used as an early prognostic marker in recurrent cervical cancer patients (50). Patients with greater HMGB1 expression have a higher rate of hrHPV infection recurrence than those with weak HMGB1 expression (51). Mechanisms employed by HMGB1 to induce the immune tolerance to hrHPV infection are proposed to be as follows: HMGB1 suppresses the human immune function by upregulating Tregs and promoting IL-10 production. It also inhibits the function of T cells by downregulating $\mathrm{NF}-\kappa \mathrm{B}$ signaling and polarizing Th1 cells to Th2 cells. HMGB1 is becoming a useful biomarker for the evaluation of hrHPV infection persistency and CIN progression (52).

p $16^{\text {INK4a }}$ expression is induced by the integration of hrHPV DNA into the host genome; it is the cyclin-dependent kinase inhibitor and is overexpressed in CIN (53). p16 ${ }^{\mathrm{INK} 4 \mathrm{a}}$ 
expression has a positive correlation with the CIN grade, and could be used to discriminate between different CIN grades in cervical biopsies.

Generally, hrHPV L1 capsid protein is expressed in every stage of CIN, with a negative association with the CIN grades (54). The abortion of hrHPV cycles in HSIL results in the L1 capsid protein being non-detectable (55). From the immunological perspective, hrHPV L1 is the inducer for the $\mathrm{CD}^{+}$and $\mathrm{CD}^{+} \mathrm{T}$ cell response against $\mathrm{hrHPV}$ infection (56). Therefore, reduced L1 capsid expression is a significant mechanism for the immune evasion of higher grade lesions.

\section{Preventive and therapeutic HPV vaccines}

The majority of clinical trials concerning HPV-targeted therapies are based on eliciting cell-mediated immune responses with vaccines. Finding a target to generate a specific immune response is the strategy for the treatment of hrHPV infection, as well as cervical cancer (57). The preventive vaccination against HPV16 and 18 has now become widely used (58). Antigen-specific immunotherapy is one of the effective methods to elicit immune responses to hrHPV. Listeria monocytogenes (LM) has been used to eliminate palpable, vascularized tumors in several mouse models due to its ability to generate $\mathrm{CD} 8^{+}$tumor-infiltrating lymphocytes. Two vaccines, LM-LLO-E7 and LM-ActA-E7, have been created by a truncated listeriolysin O (LLO) fused to E7 and a fragment of the ActA protein fused to E7, respectively. These vaccines overcome central tolerance by expanding low avidity $\mathrm{CD}^{+} \mathrm{T}$ cells specific for E7 (59). A novel fusion protein, HPV16 E7-HBcAg-Hsp65 (VR111), which could elicit an E7-specific $\mathrm{CD}^{+} \mathrm{T}$ cell response, is a novel potential preventive vaccine (60).

Therapeutic vaccines aim to clear hrHPV infections and the hrHPV-related cervical lesions, and are mainly targeted to oncoproteins E6 and E7, the only viral proteins expressed in cervical cancer and precursor lesions. Previously, E6/E7 oncoproteins were used as the target of the therapeutic vaccine, which could increase the lesion-infiltrating $\mathrm{CD}^{+}$and $\mathrm{CD} 8^{+}$ $\mathrm{T}$ cells in the intraepithelial neoplasia lesions (61). In a study, a novel HPV16 E6 and E7 gene plasmid containing oligomannose liposomes (OML-HPV) was been generated for the immunotherapy of cervical cancer. With the stimulation of OML-HPV, HPV16 E6-specific CTLs could be generated from peripheral blood mononuclear cells for $\mathrm{HPV} 16^{+}$cervical carcinoma patients (62).

During the analysis of the HPV vaccines, it has been found that the DC-based HPV vaccine is a promising tool to prevent and treat hrHPV infection, as well as cervical cancer. DCs generated from peripheral blood monocytes with IL-4 and granulocyte-macrophage colony-stimulating factor were treated with HPV16 mE7, which showed the significantly increased expression of co-stimulatory molecules CD80 and $\mathrm{CD} 40$, and the marked production of IL-12p70 and IFN- $\gamma$. This subset of DCs could upregulate E7-specific CD8 ${ }^{+}$ $\mathrm{T}$ cell responses in patients with hrHPV-associated cervical cancer (63). HPV16 E7 polypeptide is another DC-based HPV vaccine. DC loaded with hrHPV16 E7 polypeptide in combination with CpG-ODN2006 also showed distinguished immunotherapeutic activity. When SCID mice were inoculated with such antigen-loaded DCs, they exhibit decreased tumor size, increased IgG and IFN- $\gamma$ levels and increased CTL activity (64). Another way to activate the DC immune response is via genetic modification. A genetically-modified $\mathrm{DC}$ vaccine expressing HPV16 E6/E7 fusion oncoproteins was able elicit the action of E6/E7-specific CTLs against cervical carcinoma CaSki cells and induce their apoptosis. These elicited specific protective immunity functions by the HPV-16 E6/E7 fusion protein produced an effective approach against cervical cancer cell growth (65).

Different types of vaccine have been investigated; one of the most important mechanisms for their utilization is based on their initiating the damaged immune response by hrHPV. Schematic representation of this has been presented in Fig. 2 .

\section{Conclusion}

The present review investigated the association between hrHPV and cervical cancer. The immune system plays an important role from HPV infection to CIN and from CIN to cervical cancer. A compromised immune response is the prerequisite for disease progression. One unique feature of HPV infection is that it can affect the immune system in such as way that it presents a much more tolerant state, which facilitates persistent hrHPV infection and cervical lesion progression. The counteraction of hrHPV infection by vaccines is an important tool to stop the course of the infection or oncogenesis. To date, numerous preventive and therapeutic vaccines have been discovered, with the aim of enhancing the damaged immune response to clear the virus and the tumor cells.

\section{References}

1. Parkin DM and Bray F: Chapter 2: The burden of HPV-related cancers. Vaccine 24 (Suppl 3): 3/11-25, 2006.

2. Almonte M, Albero G, Molano M, et al: Risk factors for human papillomavirus exposure and co factors for cervical cancer in Latin America and the Caribbean. Vaccine 26: L16-L36, 2008.

3. Deligeoroglou E, Giannouli A, Athanasopoulos N, et al: HPV infection: Immunological aspects and their utility in future therapy. Infect Dis Obstet Gynecol 2013: 540850, 2013.

4. Bernard HU, Burk RD, Chen Z, et al: Classification of papillomaviruses (PVs) based on $189 \mathrm{PV}$ types and proposal of taxonomic amendments. Virology 401: 70-79, 2010.

5. Bouvard V, Baan R, Straif K, et al: A review of human carcinogens-Part B: Biological agents. Lancet Oncol 10: 321-322, 2009.

6. Coutlée F, Rouleau D, Petignat P, et al: Enhanced detection and typing of human papillomavirus (HPV) DNA in anogenital samples with PGMY primers and the Linear array HPV genotyping test. J Clin Microbiol 44: 1998-2006, 2006.

7. Smith JS, Lindsay L, Hoots B, et al: Human papillomavirus type distribution in invasive cervical cancer and high-grade cervical lesions: A meta-analysis update. Int J Cancer 121: 621-632, 2007.

8. Cerqueira C, Liu Y, Kühling L, et al: Heparin increases the infectivity of Human Papillomavirus type 16 independent of cell surface proteoglycans and induces L1 epitope exposure. Cell Microbiol 15: 1818-1836, 2013.

9. Surviladze Z, Dziduszko A and Ozbun MA: Essential roles for soluble virion-associated heparan sulfonated proteoglycans and growth factors in human papillomavirus infections. PLoS Pathog 8: e1002519, 2012.

10. Raff AB, Woodham AW, Raff LM, et al: The evolving field of human papillomavirus receptor research: A review of binding and entry. J Virol 87: 6062-6072, 2013.

11. Horvath CA, Boulet GA, Renoux VM, Delvenne PO and Bogers JP: Mechanisms of cell entry by human papillomaviruses: An overview. Virol J 7: 11, 2010. 
12. Asiaf A, Ahmad ST, Mohammad SO and Zargar MA: Review of the current knowledge on the epidemiology, pathogenesis and prevention of human papillomavirus infection. Eur J Cancer Prev 23: 206-224, 2014.

13. Venuti A, Paolini F, Nasir L, et al: Papillomavirus E5: the smallest oncoprotein with many functions. Mol Cancer 10: 140, 2011.

14. Campo MS, Graham SV, Cortese MS, et al: HPV-16 E5 down-regulates expression of surface HLA class I and reduces recognition by CD8 T cells. Virology 407: 137-142, 2010.

15. Faridi R, Zahra A, Khan K and Idrees M: Oncogenic potential of human papillomavirus (HPV) and its relation with cervical cancer. Virol J 8: 269, 2011.

16. Zuna RE, Allen RA, Moore WE, Mattu R and Dunn ST: Comparison of human papillomavirus genotypes in high-grade squamous intraepithelial lesions and invasive cervical carcinoma: Evidence for differences in biologic potential of precursor lesions. Mod Pathol 17: 1314-1322, 2004.

17. Hasan UA, Zannetti C, Parroche P, et al: The human papillomavirus type 16 E7 oncoprotein induces a transcriptional repressor complex on the Toll-like receptor 9 promoter. J Exp Med 210: 1369-1387, 2013.

18. Kim H, Kwon B and Sin JI: Combined stimulation of IL-2 and 4-1BB receptors augments the antitumor activity of E7 DNA vaccines by increasing Ag-specific CTL responses. PLoS One 8: e83765, 2013

19. Bedoya AM, Jaramillo R, Baena A, et al: Location and density of immune cells in precursor lesions and cervical cancer. Cancer Microenviron, Jan 31, 2012 (Epub ahead of print).

20. Stanley MA and Sterling JC: Host responses to infection with human papillomavirus. Curr Probl Dermatol 45: 58-74, 2014.

21. Crosbie EJ, Einstein MH, Franceschi S and Kitchener HC: Human papillomavirus and cervical cancer. Lancet 382 889-899, 2013

22. Piersma SJ: Immunosuppressive tumor microenvironment in cervical cancer patients. Cancer Microenviron 4: 361-375, 2011.

23. Alves DB, Tozetti IA, Gatto FA, et al: CD4 and CD8 T lymphocytes and NK cells in the stroma of the uterine cervix of women infected with human papillomavirus. Rev Soc Bras Med Trop 43: 425-429, 2010.

24. Feller L, Wood NH, Khammissa RA, et al: HPV modulation of host immune responses. SADJ 65: 266-268, 2010.

25. Le Borgne M, Etchart N, Goubier A, et al: Dendritic cells rapidly recruited into epithelial tissues via CCR6/CCL20 are responsible for $\mathrm{CD}^{+} \mathrm{T}$ cell cross priming in vivo. Immunity 24: 191-201, 2006.

26. Hasan U: Human papillomavirus (HPV) deregulation of Toll-like receptor 9. Oncoimmunology 3: e27257, 2014.

27. Zhang Y, Yang H, Barnie PA, et al: The expression of Toll-like receptor 8 and its relationship with VEGF and $\mathrm{Bcl}-2$ in cervical cancer. Int J Med Sci 11: 608-613, 2014.

28. Wang Y, Weng Y, Shi Y, et al: Expression and Functional Analysis of Toll-like Receptor 4 in Human Cervical Carcinoma. J Membr Biol 247: 591-599, 2014.

29. Hammes LS, Tekmal RR, Naud P, Edelweiss MI, et al: Macrophages, inflammation and risk of cervical intraepithelial neoplasia (CIN) progression-clinicopathological correlation Gynecologic Oncology 105: 157-165, 2007.

30. Lepique AP, Daghastanli KR, Cuccovia IM and Villa LL: HPV16 tumor associated macrophages suppress antitumor $\mathrm{T}$ cell responses. Clinical Cancer Research 15: 4391-4400, 2009.

31. Garcia-Iglesias T, Del Toro-Arreola A, Albarran-Somoza B, et al: Low NKp30, NKp46 and NKG2D expression and reduced cytotoxic activity on NK cells in cervical cancer and precursor lesions. BMC Cancer 9: 186, 2009.

32. Jimenez-Perez MI, Jave-Suarez LF, Ortiz-Lazareno PC, et al: Cervical cancer cell lines expressing NKG2D-ligands are able to down-modulate the NKG2D receptor on NKL cells with functional implications. BMC Immunol 13: 7, 2012.

33. Kobayashi A, Weinberg V, Darragh T and Smith-McCune K Evolving immunosuppressive microenvironment during human cervical carcinogenesis. Mucosal Immunol 1: 412-420, 2008.

34. Bais AG, Beckmann I, Lindemans J, et al: A shift to a peripheral Th2-type cytokine pattern during the carcinogenesis of cervical cancer becomes manifest in CIN/III lesions. J Clin Pathol 58 1096-1100, 2005.

35. Scott ME, Shvetsov YB, Thompson PJ, et al: Cervical cytokines and clearance of incident human papillomavirus infection: Hawaii HPV cohort study. Int J Cancer 133: 1187-1196, 2013.
36. Peghini BC, Abdalla DR, Barcelos AC, Teodoro L, Murta EF and Michelin MA: Local cytokine profiles of patients with cervical intraepithelial and invasive neoplasia. Hum Immunol 73 920-926, 2012

37. Lee YS, Lee CW, Song MJ, et al: Cell-mediated immune response to human papillomavirus 16 E7 peptide pools in patients with cervical neoplasia. Acta Obstet Gynecol Scand 90: 1350-1356, 2011

38. Sheu BC, Chang WC, Lin HH, Chow SN and Huang SC: Immune concept of human papillomaviruses and related antigens in local cancer milieu of human cervical neoplasia. J Obstet Gynaecol Res 33: 103-113, 2007.

39. Iijima N, Goodwin EC, Dimaio D and Iwasaki A: High-risk human papillomavirus E6 inhibits monocyte differentiation to Langerhans cells. Virology 444: 257-262, 2013.

40. Strickler HD, Martinson J, Desai S, et al: The relation of plasmacytoid dendritic cells (pDCs) and regulatory T-cells (Tregs) with HPV persistence in HIV-infected and HIV-uninfected women. Viral Immunol 27: 20-25, 2014

41. Yang W, Song Y, Lu YL, Sun JZ and Wang HW: Increased expression of programmed death (PD)-1 and its ligand PD-L1 correlates with impaired cell-mediated immunity in high-risk human papillomavirus-related cervical intraepithelial neoplasia. Immunology 139: 513-522, 2013.

42. Adurthi S, Krishna S, Mukherjee G, et al: Regulatory T cells in a spectrum of HPV-induced cervical lesions: Cervicitis, cervical intraepithelial neoplasia andsquamous cell carcinoma. Am J Reprod Immunol 60: 55-65, 2008.

43. de Vos van Steenwijk PJ, Piersma SJ, Welters MJ, et al: Surgery followed by persistence of high-grade squamous intraepithelial lesions is associated with the induction of a dysfunctional HPV16-specific T-cell response. Clin Cancer Res 14: 7188-7195, 2008.

44. Ali KS, Ali HY and Jubrael JM: Concentration levels of IL-10 and TNF $\alpha$ cytokines in patients with human papilloma virus (HPV) $\mathrm{DNA}^{+}$and DNA-cervical lesions. J Immunotoxicol 9: 168-172, 2012.

45. Bermudez-Morales VH, Gutierrez LX, Alcocer-Gonzalez JM, Burguete $\mathrm{A}$ and Madrid-Marina V: Correlation between IL-10 gene expression and HPV infection in cervical cancer: a mechanism for immune response escape. Cancer Invest 26 1037-1043, 2008

46. Bolpetti A, Silva JS, Villa LL and Lepique AP: Interleukin-10 production by tumor infiltrating macrophages plays a role in Human Papillomavirus 16 tumor growth. BMC Immunol 11: 27, 2010.

47. Bermudez-Morales VH, Peralta-Zaragoza O, Alcocer Gonzalez JM, Moreno J and Madrid-Marina V: IL-10 expression is regulated by HPV E2 protein in cervical cancer cells. Mol Med Rep 4: 369-375, 2011.

48. Koshiol J, Sklavos M, Wentzensen N, et al: Evaluation of a multiplex panel of immune-related markers in cervical secretions: a methodologic study. Int J Cancer 134: 411-425, 2014.

49. Zeng C, Yao Y, Jie W, et al: Up-regulation of Foxp3 participates in progression of cervical cancer. Cancer Immunol Immunother 62: 481-487, 2013

50. Sheng $X$, Du X, Zhang X, et al: Clinical value of serum HMGB1 levels in early detection of recurrent squamous cell carcinoma of uterine cervix: Comparison with serum SCCA, CYFRA21-1 and CEA levels. Croat Med J 50: 455-464, 2009.

51. Huang LF, Yao YM, Zhang LT, Dong N, Yu Y and Sheng ZY: The effect of high-mobility group box 1 protein on activity of regulatory T cells after thermal injury in rats. Shock 31: 322-329, 2009.

52. Pang X, Zhang Y, Wei H, et al: Expression and effects of high-mobility group box 1 in cervical cancer. Int J Mol Sci 15: 8699-8712, 2014.

53. Halloush RA, Akpolat I, Jim Zhai Q, Schwartz MR and Mody DR: Comparison of ProEx C with p16INK4a and Ki-67 immunohistochemical staining of cell blocks prepared from residual liquid-based cervicovaginal material: A pilot study. Cancer 114: 474-480, 2008

54. Izadi-Mood N, Sarmadi S, Eftekhar Z, Jahanteegh HA and Sanii S: Immunohistochemical expression of p16 and HPV L1 capsid proteins as predictive markers in cervical lesions. Arch Gynecol Obstet 289: 1287-1292, 2014

55. Lee SJ, Lee AW, Kang CS, et al: Clinicopathological implications of human papilloma virus (HPV) L1 capsid protein immunoreactivity in HPV16-positive cervical cytology. Int J Med Sci 11: 80-86, 2013. 
56. Pinto LA, Edwards J, Castle PE, et al: Cellular immune responses to human papillomavirus (HPV)-16 L1 in healthy volunteers immunized with recombinant HPV-16 L1 virus-like particles. J Infect Dis 188: 327-338, 2003.

57. Chen Z, Kamath P, Zhang S, St John L, Adler-Storthz K and Shillitoe EJ: Effects on tumor cells of ribozymes that cleave the RNA transcripts of human papillomavirus type 18. Cancer Gene Ther 3: 18-23, 1996.

58. Knoff J, Yang B, Hung CF and Wu TC: Cervical cancer: development of targeted therapies beyond molecular pathogenesis. Curr Obstet Gynecol Rep 3: 18-32, 2014.

59. Souders NC, Sewell DA, Pan ZK, et al: Listeria-based vaccines can overcome tolerance by expanding low avidity $\mathrm{CD} 8^{+} \mathrm{T}$ cells capable of eradicating a solid tumor in a transgenic mouse model of cancer. Cancer Immu 7: 2, 2007.

60. Zhou CM, Zhang GX and Ma XX: Characterization and evaluation of the immune responses elicited by a novel human papillomavirus (HPV) therapeutic vaccine: HPV 16E7-HBcAg-Hsp65 fusion protein. J Virol Methods 197: 1-6, 2014.

61. Davidson EJ, Boswell CM, Sehr P, et al: Immunological and clinical responses in women with vulval intraepithelial neoplasia vaccinated with a vaccinia virus encoding human papillomavirus 16/18 oncoproteins. Cancer Res 63: 6032-6041, 2003.
62. Mizuuchi M,Hirohashi Y, Torigoe T, et al: Novel oligomannose liposome-DNA complex DNA vaccination efficiently evokes anti-HPV E6 and E7 CTL responses. Exp Mol Pathol 92: 185-190, 2012.

63. Wang YT, Li W, Liu Q, Guan X and Hu J: Dendritic cells treated with HPV16mE7 in a three-dimensional model promote the secretion of IL-12p70 and IFN- $\gamma$. Exp Mol Pathol 91: 325-330, 2011.

64. Wang HL, Xu H, Lu WH, Zhu L, Yu YH and Hong FZ: In vitro and in vivo evaluations of human papillomavirus type 16 (HPV16)-derived peptide-loaded dendritic cells (DCs) with a $\mathrm{CpG}$ oligodeoxynucleotide (CpG-ODN) adjuvant as tumor vaccines for immunotherapy of cervical cancer. Arch Gynecol Obstet 289: 155-162, 2014

65. Wu XM, Liu X, Jiao QF, et al: Cytotoxic T Lymphocytes elicited by dendritic cell-targeted delivery of human papillomavirus Type-16 E6/E7 fusion gene exert lethal effects on CaSki cells. Asian Pac J Cancer Prev 15: 2447-2451, 2014. 